\title{
Newly discovered polymorphism associated with prostate cancer risk in African-Americans
}

\section{'This find ing emphasizes the importance of ancestry in studying genetic s.'}

Researchers seeking to confirm that results of previous studies linking genetic variations to prostate cancer risk in Caucasians were al so valid in African-Americans have discovered an additional, independent genetic variant that was also significantly associated with cancer risk in this population.

Prostate cancer is a cancer of the prostategland of the male reproductive system that tends to affect men over 50 years of age. It is the second most common cause of cancer death and the most common malignancy in men. Rates of prostate cancer vary quite considerably between ethnic groups, with African-American men exhibiting the highest and Asian men the lowest rates worldwide. Generally the rates of incidence increase from east to west across the world, with America having the highest frequency of the disease. Screening for prostate cancer generally begins after the age of 50 , but it has been suggested that this should be started as early as 35 , especially in high-risk groups such as African-Americans.

Previous research has pinpointed several genetic variants found in a small area on chromosome 8 that seem to be associated with increased risk of prostate cancer in Caucasian men. Thescientistsinvolved with this study aimed to confirm whether these results were also valid in a group of African-American men. Approximately 100 linked and unlinked markers were genotyped in a group of 490 African-Americans previously diagnosed with prostate cancer, as well as a control group of 567 ethnically matched men without cancer.

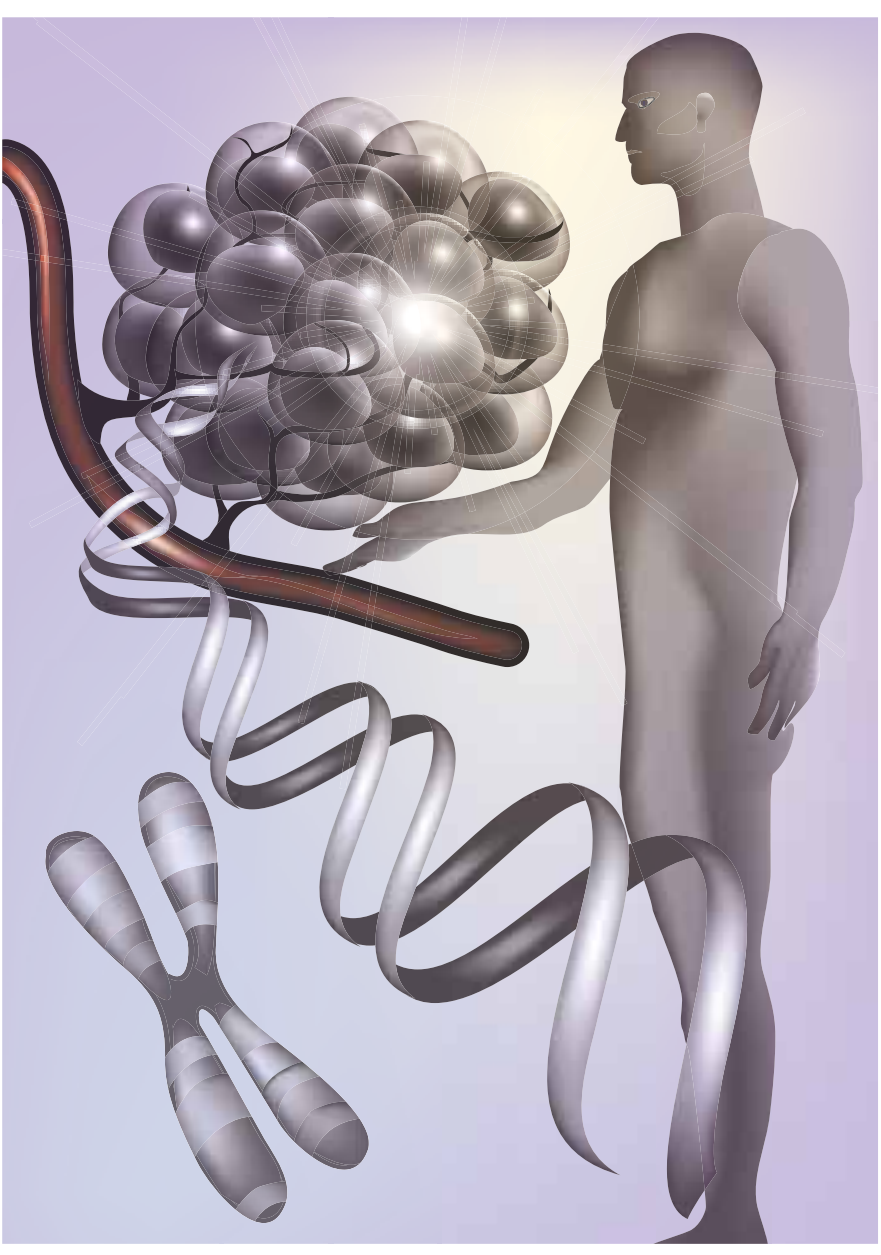

The researchers managed to replicate the association found between one of the previously isolated polymorphisms (rs16901979), and risk of developing the condition, as well as discovering a new variant (rs7008482) located within a gene associated with $D N A$ replication, repair and recombination al so located in the same area of chromosome 8. It is thought that mutations in this gene might lead to an inherited predisposition to genetic instability and a higher risk of developing cancer. Rick Kittles, one of the study authors, explained: "T his finding emphasizes the importance of ancestry in studying genetics. Previous studies led us to one specific region of chromosome 8 . Then this approach - which took advantage of genetic differences among African-American men, who are at very high risk for this type of cancer - led us to a different locus within that region and directly to a gene of interest".

'The prevalence of prostate cancer is a lmost two fold higheramong

African-Americans compared with European Americans'

The results of this study are the most recent in a group of studies all isolating this relatively small area of chromosome 8 (8q24) as important in determining genetic risk factors for prostate cancer. The researchers are now focusing their efforts closely on studying this region and the function of the genes within it. This study illustrates the importance of taking ethnic background into consideration when planning and executing such research. "Since African-Americansvary significantly in genetic ancestral proportions and the prevalence of prostate cancer is almost twofold higher among African-Americans compared to European Americans," commented Rick Kittles, "the use of ancestry-informative markers for association with prostate cancer is quite powerful".

Source: Robbins C, Torres JB, H ooker S et al.: Confirmation study of prostatecancer risk variants at 8q24 in African-Americans identifies a novel risk locus. Genome Res. Published 0 ctober 31, 2007, 10.1101/gr.6782707 


\section{in brief...}

Masking epilepsy by combining two epilepsy genes.

Glasscock E, Qian J, Yoo JW, Noebels JL: Nat. Neurosci. (2007) (Epub ahead of print).

Researchers in this study created a mouse model of spontaneously occurring epilepsy by inducing two previously described ion channel mutations associated with the condition. The phenotypes of these variants were known to have overlapping subcellular localizations, as well as expressing opposing excitability defects. One of the mutations, in the Kcnal gene, known to cause severe seizures and occasionally death, was masked by another mutation in the Cacnala gene, which is responsible for causing absence epilepsy; the converse was also found to be true. Additionally, intermediate excitability phenotypes were identified at the axonal and network levels. This work illustrates the extent to which such protective interactions may alter the phenotype expressed by the individual in question. It also highlights the need for intensive profiling of this group of genes in order to improve the accuracy of future genetic risk assessment for epilepsy.

Mutation in glycerol-3-phosphate dehydrogenase 1 like gene (GPD1-L) decreases cardiac $\mathrm{Na}^{+}$current and causes inherited arrhythmias.

London $\mathrm{B}, \mathrm{M}$ ichalec $\mathrm{M}$, Mehdi $\mathrm{H}$ et al.: Circulation (2007) (Epub ahead of print). This study investigated the genetics of the rare, autosomal dominant and male predominant heart condition known as Brugada syndrome. Individuals with this condition suffer from ventricular fibrillation, sometimes causing fainting and cardiac arrest. $20 \%$ of cases of Brugada syndrome are caused by mutations in the SCN5A gene on chromosome 3, most of which reduce inward current of sodium. Positional cloning was used previously to isolate an additional locus (not SCN5A), also on chromosome 3 , in a large family with the condition. In this study, direct sequencing was used to identify a polymorphism (A280V) in the gene GPD1-L (whose protein is abundant in the heart) that was found to be present in all the individuals with the condition. Additionally, the mutation was not found in a control group of over 500. Coexpresison of the GPD1-L mutation with SCN5A in HEK cells lowered inward flowing sodium currents by around $50 \%$, as well as reducing $\mathrm{SCN} 5 \mathrm{~A}$ cell surface expression. This research has uncovered a novel genetic cause of Brugada syndrome, as well as providing valuable information about genetic control of sodium movement in these cells.

\section{Children with Alzheimer's gene variant display poor spatial learning and memory}

Scientists have discovered that children who possess an allele of the Apolipoprotein E (APOE) gene, commonly associated with increased risk of Alzheimer's disease, show reduced cognitive ability at an early age.

The APOE gene produces proteins that are instrumental in redistribution and metabolism of cholesterol and lipoproteins. It has three main alleles, APOE2, 3 and 4.

"One of our questions has been is this a risk that only happens with age, or is it already - early on - the cause of differences in performance".

APOE4 has previously been shown to be a strong risk factor for Alzheimer's disease, although not a determinant, and $40-65 \%$ of all Alzheimer's patients have at least one copy of this allele. APOE2 and 3 do not appear to be associated with the condition, although there is some previous evidence that APOE2 may have a protective effect.

The researchers involved with this study hypothesized that the cognitive problems associated with APOE4 may affect carriers at a much earlier age than previously anticipated. "O ne of our questions has been is this a risk that only happens with age, or is it already - early on - the cause of differences in performance," commented study coauthor Jacob Raber, "T his study suggests there already are cognitive differences very early on in life".

They tested a group of 55 healthy 7- 10 year-old children ( 25 girls and 30 boys) for the presence of the APOE4 allele. $0 f$ this group, six boys and eight girls carried the allele and the others who did not acted as controls. After determining their genotype, the cognitive abilities of the children were assessed using a combination of paper and computer based tests including a program called 'M emory Island' that tests spatial memory.

It was found that children with the APOE4 allele did significantly worse than children who were not carriers in both spatial learning and memory tests. This is an important discovery about the effects of this allele on cognitive development and ability, and implies that susceptibility to the decline seen in elderly carriers is present from an early age. It also introduces the possibility that therapeutic interventions to prevent such decline may be possible at a much younger age.

Sources: Acevedo SF, Raber J: ApoE4 affects spatial learning and memory in children. Program No. 422.6/FFF28. Society for Neuroscience, 2007. 2007N euroscience M eeting Planner, San Diego, CA, USA. www.sfn.org/am2007/

\section{About the Bulletin Board}

The Bulletin Board highlights some of the most important events and launches in pharmacogenomics. The editorial team welcomes suggestions for timely, relevant items. If you have newsworthy information, please contact:

Shreeya Nanda, Commissioning Editor,

Pharmacogenomics, Future Medicine Ltd,

Unitec House, 2 Albert Place,

London, N3 1QB, UK

s.nanda@futuremedicine.com

Tel.: +44 (0)20 83716090 Fax: +44 (0)20 83432313 


\section{C reation of hard-drinking and lightweight Drosophila sheds light on genetics of alcohol tolerance}

Scientists have adopted a novel approach to studying the genetics of alcohol tolerance by selectively breeding two strains of lightweight and hard-drinking D rosophila and comparing their genetics with thehope of pinpointing genesthat may beassociated with variation in human alcohol tolerance.

The researchers involved in the study artificially selected for al cohol sensitivity by exposing flies to alcohol through a device called an inebriometer. This involved passing alcohol vapour through a long tube with a series of slanted platforms for flies to perch on. Flies were initially introduced at the top of the tube and as the alcohol started to affect the flies they gradually fell from platform to platform until reaching the bottom of the tube.

The flies were divided in this way into extreme lightweights and those that had the longest exposure to alcohol before reaching the bottom. These groups were then bred for 35 generations to create lines of flies that were either highly sensitive (only lasting for around $1 \mathrm{~min}$ in the inebriometer) or resistant (lasting a lengthy $18 \mathrm{~min}$ before falling to the bottom of the tube) to ethanol exposure.

"We wanted to find the genetic factors that changed when flies became more sensitive or more resista nt to a lc ohol"

W holegenome expression analysis was then carried out on the different lines of flies and the results compared to try and isolate genes with altered expression indicating potential involvement in sensitivity to alcohol. "We wanted to find the genetic factors that changed when flies became more sensitive or more resistant to alcohol, knowing that genes that undergo changes are potential candidate genes for mediating sensitivity," commented Robert Anholt, one of the researchers involved in the study.

The results indicated that over 1600 genes displayed altered expression. of these, 35 candidate genes were selected to betested in more detail and 32 were found to be involved in alcohol sensitivity. The researchers hope to find the human orthologues for these genes which, due to evolutionary conservation of function, are also likely to be involved in alcohol sensitivity and processing in humans. It is hoped that research such as this will throw light on the genetics behind alcohol susceptibility and conditions such as alcoholism and will help doctors predict those whose health is likely to be most at risk from excessive consumption.

Source: M orozova T V, Anholt RR, M ackay TF: Phenotypic and transcriptional response to selection for alcohol sensitivity in D rosophila melanogaster. Genome Biol. 8(10), R231 (2007).

\section{Tumor suppressor gene associated with drug resistance in neuroblastoma cells}

Researchers have discovered a new role for the p53 tumor suppressor gene in controlling tumor response to neuroblastoma chemotherapy drugs.

"Our results provide definitive evidence of a role for p53 as a gene which dictates drug sensitivity in neuroblastoma,"

$\mathrm{N}$ euroblastoma is the most common childhood, tumor-causing, non-brainrelated cancer, accounting for $6-10 \%$ of all childhood cancers. It mainly affects younger children, with fewer than $10 \%$ of cases occurring in children over the age of 10 years. It affects the sympathetic nervous system, most often causing the development of solid tumors in the adrenal glands. The p53 gene produces a protein that is involved in regulation of cell division. M utations in this gene have been previously associated with many different cancers, leading to it being known as a tumor suppressor gene in its normal, unmutated state.

N euroblastoma often goes into remission after chemotherapy treatment only to return at a later stage. Problems can occur with further chemotherapy treatment, including increased side effects and drug resistance. Previous work showed heavy metal-selected neuroblastoma cells to develop a wide multidrug resistance (M D R) phenotype not associated with formerly observed M D R-linked genes. p53 mutations are rarely seen at diagnosis of neuroblastoma cases, but have been observed to arise after treatment with chemotherapy in these cells. The researchers in this study used different cell lines to investigate the effect of mutations in the p53 gene on sensitivity to chemotherapy drugs used to combat this cancer. p53 deficient cell lines were generated from neuroblastoma cell lines containing normal copies of the 553 gene. The mutant cell lines with inactivated p53 developed resistance to a number of different chemotherapy drugs, as opposed to the wild type cell lines in which p53 was fully functional. "O ur results provide definitive evidence of a role for p53 as a gene which dictates drug sensitivity in neuroblastoma," confimed lead researcher and author Chengyuan Xue. Headded the caution that: "Further research has found that p53 does not have one defined function in cancer susceptibility to treatment. In some cancer cell types it does not act as a drug sensitivity gene. It is therefore important to assess the clinical effect of p53 mutations in different cancers in a tissue context". Source: Xue C, H aber M , Flemming C et al.: p53 determines multidrug sensitivity of childhood neuroblastoma. Cancer Res. 67(21),

10351-10360 (2007). 